\title{
The Near Future of Children's Robotics
}

\section{Vicky Charisi}

UCL, Institute of Education

London, UK

v.charisi@utwente.nl

\section{Alyssa M. Alcorn}

Centre for Research in Autism

and Education

UCL Institute of Education

London, UK

a.alcorn@ucl.ac.uk

\section{James Kennedy}

Disney Research

Los Angeles, USA

james.kennedy@

disneyresearch.com

\author{
Wafa Johal \\ CHILI / LSRO Labs \\ EPFL \\ Lausanne, Switzerland \\ wafa.johal@epfl.ch \\ Paul Baxter \\ Lincoln Centre for Autonomous \\ Systems \\ School of Computer Science \\ University of Lincoln, UK \\ pbaxter@lincoln.ac.uk

\section{Chronis Kynigos} \\ Educational Technology Lab \\ NKUA \\ Athens, Greece \\ kynigos@ppp.uoa.gr
}

Permission to make digital or hard copies of part or all of this work for personal or classroom use is granted without fee provided that copies are not made or distributed for profit or commercial advantage and that copies bear this notice and the full citation on the first page. Copyrights for third-party components of this work must be honored. For all other uses, contact the Owner/Author.

IDC '18, June 19-22, 2018, Trondheim, Norway (C) 2018 Copyright is held by the owner/author(s). ACM ISBN 978-1-4503-5152-2/18/06.

https://doi.org/10.1145/3202185.3205868

\section{Abstract}

Robotics is a multidisciplinary and highly innovative field. Recently, multiple and often minimally connected sub-communities of child-robot interaction have started to emerge, variously focusing on the design issues, engineering, and applications of robotic platforms and toolkits. Despite increasing public interest in robots, including robots for children, child-robot interaction research remains highly fragmented and lacks regular cross-disciplinary venues for discussion and dissemination. This workshop will bring together researchers with diverse scientific backgrounds. It will serve as a venue in which to reflect on the current circumstances in which child-robot research is conducted, articulate emerging and "near future" challenges, and discuss actions and tools with which to meet those challenges and consolidate the field.

\section{Author Keywords}

Children's robotics; robots; Child-robot interaction;

interaction design; Educational robotics; Ethics; design.

\section{ACM Classification Keywords}

H.1.2 [User/Machine Systems]: Human factors; H.5.m Information interfaces and presentation (e.g., $\mathrm{HCI}$ ): Miscellaneous; K.3.0 [Computers and Education]: General

\section{Background}

Child-robot interaction (CRI) is a rapidly-expanding but still fragmented area of research. Projects and lines of research that sound similar have sometimes developed out of different "host" disciplines, and have different theoretical or epistemological underpinnings. Robots have been increasingly represented in the Interaction 
Design and Children (IDC) community e.g. [2, 14, 21], but work with children and robotics has also been conducted in more engineering-based contexts for at least two decades $[13,17,23]$, within developmental psychology $[20,24]$, in educational technology and design $[5,10,18]$ as well as in industry. Just as mainstream human-computer interaction communities often do not address issues specific to children's technologies, so too are the needs of CRI not fully met by broad research communities for human-robot interaction. There are not yet dedicated conferences or publications in which the diverse group of researchers working on children and robots may exchange ideas and consolidate individual CRI projects into Children's Robotics, as a field-or to collectively articulate their concerns and priorities.

Regardless of their "host" disciplines and precise goals, all CRI researchers are currently operating in a climate of high expectations and overwhelming public interest in robotics. New popular articles, videos, and opinion pieces appear daily in many languages e.g. [3] actively shaping views and expectations about robots, often with limited reference to robotics research. Robots have captured people's imaginations, and appear increasingly acceptable and desirable-including for child users [9]. At the same time, the number of available robots and their technical capabilities are growing and becoming more easily accessible for the general public. For example, robots are perceived to offer a playful and tangible way for children to engage with technology and to develop engineering concepts through programming, even in early childhood e.g. [22]. As a result, there is an increasing interest from schools and informal education centres to include robots in their curricula as learning tools.
The other side of the positive public momentum around robotics is that potential problems are also gathering momentum. For example, recent hacks and security vulnerabilities discovered in internet-connected "smart" toys, some robot-like, have highlighted the substantial work yet to do on ethics and practicalities of data handling-not to mention children's privacy, dignity, and rights as technology users [12]. These issues from current technologies "in the wild" have major implications for child-robot interaction now and in the near future, especially when designing interactions meant to be personal, social, or sustained over time. Ethical incidents highlight another potential challenge: a backlash in which potential users could pivot from over-trust to rejection of apparently autonomous technologies, including robots. Teachers, for example, have started to express concerns regarding emerging ethical considerations such as privacy and the impact of robots on child development [19]. Technology research cannot divorce itself from public views, positive or negative. Especially because CRI researchers request access to children, families, homes, and schools as a part of our research, people's opinions and preconceptions of robotics matter and directly affect the research that do.

The current conjunction of public appetite for children's robotics and robots' shifting capabilities suggests that the climate in which CRI researchers operate is facing a period of rapid change. Looking five years forward, the "near future" of CRI may be different, both in terms of what is possible and what child users (and adult gatekeepers) want, expect, or find acceptable from robots. Recent and in-progress research allows us to identify some of these changes at work, and to try to 
predict their trajectories toward future challenges in CRI research-or toward future strengths.

Recent projects with children and robotics suggest that design partnership with end-users may be an area of future opportunity and growth for CRI research. Several current projects, for example, have sought to evaluate the impact of robots in therapy for autistic children, from a clinical perspective. Ongoing work, (see http://bit.ly/2BnhJZ6 for a preliminary project draft) aims to produce a whitebook with ethical guidelines for the use of robots with children in such contexts. In the same line, technically-focused and autism-focused teams, that develop a robot-assisted teaching program for facial emotions $[1,7]$, conducted interviews with over 40 autism educators in the UK and Serbia (in preparation), which have revealed a high degree of openness and optimism about the potential use of humanoid robots in school. Both the interviews and studies suggest that there is scope and appetite for increasing participatory design research around robotics in education [11]. Educators' involvement in formative stages of research will be particularly important going forward, to address other potential challenges around the acceptability and feasibility of robots. In addition to the educators' involvement, the success and benefit of children's involvement in designing other technologies highlights opportunities for CRI research to further include children's views in design and development. Research has already been carried out, for example, on children's perspectives on robots' social competence [6], which revealed that children tend to ascribe social competency and agency to robots despite their awareness of robots' artificiality.
Robotics in school contexts and as educational tools is another potential area of future challenges and opportunities, and also one where users' needs and expectations will play a major role. Current educational agendas in many countries place increasing value on

teaching computational thinking and programming e.g. [8]. Multiple projects are exploring robotics as a way to give children direct experience of exercising these skills, in a way that may be engaging, visible, and immediate [14]. Deploying robots in class poses other questions however, such as the practical, interactional, or cognitive aspects of using them over time and as part of a larger curriculum. Some studies are already evaluating longer-term use, with larger sample sizes than earlier work, to further our understanding of the impact of using robots in educational settings $[4,16]$

\section{Scope of the Workshop}

The lack of dedicated venues for CRI research means that individual researchers and projects are disadvantaged in terms of anticipating future challenges and developing strategies to address them. Physically bringing together diverse researchers in CRI is a timely step towards consolidating and strengthening this fragmented field. This workshop is intended as an opportunity for reflection and synthesis, encouraging researchers to look outside their "home" disciplines and take a wider view of current research and the challenges we face now. Then it gives an opportunity to consider near-future challenges-and the tools available to approach them. Collectively, researchers can also consider to what extent the challenges may be shared across the larger humanrobot interaction (HRI) field, versus being specific to children. The planned discussion topics within this workshop will include: 
1. Reflecting on the current circumstances in which we conduct CRI research;

2. Identifying the already-emerging challenges that affect CRI research;

3. Identifying and prioritising perceived "near future" opportunities and challenges for CRI. For whom are these opportunities or challenges, and why? To what extent are they specific to children?

4. Exploring whether and how existing research tools could be applied to these challenges and opportunities, with particular attention to strengths within the IDC community, such as use of theory, participatory design, and application of research on child development and cognition;

5. Reflecting on participants' ongoing or planned CRI research and its connections to these emerging or "near future" issues.

At the end of the workshop, the goal is for attendees to have collectively articulated a list of near future challenges perceived to affect CRI research, as well as perceived opportunities for the field. Attendees will also collectively identify possible ways forward to meet these challenges or take advantage of these opportunities, drawing on the diverse backgrounds and methodologies represented across the group. Together, these priorities and strategies will form the backbone of the planned workshop outputs.

\section{Workshop Structure}

This full-day workshop is focused on discussion between participants, with short, informal presentations used at the start of the day to map out the expertise present and provide context for individuals' contributions. The structure of the day begins with reflection on current contexts and work in CRI, and then moves toward exploring and preparing for the "near future" (next 3-5 years) of research. The proposed schedule is as follows:

9:00 Introduction from the organisers

9:15 Participant introductions (mini-talks)

10:00 Small-group discussion 1: Current circumstances, emerging challenges

10:45 Coffee break

11:15 Small group discussion 2: Identifying nearfuture challenges and opportunities for CRI

12:15 Groups present discussion 2 conclusions

12:45 Lunch break

13:45 Whole group discussion: prioritizing challenges facing CRI

14:30 Small group discussion 3: Tools and strategies for meeting near-future challenges and opportunities

15:30 Coffee break

15:45 Groups present session 3 conclusions

16:15 Wrap-up and reflection from organisers and participants, planned outputs

17:00 End

Each small group session will have designated facilitators and note-takers. Organisers will provide facilitators with prompts such as news coverage, vignettes, or questions (e.g. "Could there be any nearfuture challenges related to ethics?") to help them start or focus the discussions, as needed.

\section{Outputs and follow-up}

The main output of this workshop will be a position paper building on the participants' characterization of the state of the field in CRI, identification and prioritization of near-future challenges, and proposed roadmap for how to address them. To our knowledge, 
no similar "state of the field" work has been published, and it will be a timely tool to help other researchers prepare for those challenges and maximize opportunities to use children's robotics in ethical and beneficial ways. Depending on the position papers received, organisers may pre-group researchers with similar work during the workshop (e.g. educational robotics, health and care robots) and who may share priorities and challenges. This would enable the position paper to more specifically comment on the extent to which perceived issues and opportunities are common across CRI research, versus particular to certain child user groups or applications of robotics.

We will invite workshop participants to be part of authoring the position paper, acknowledging that not all will be able or willing to do so. All accepted position papers will be compiled into a workshop proceedings archived on arXiv. In parallel, we will explore participants' level of interest in proposing a special journal issue on CRI, in a children's technology or human-robot interaction journal.

\section{Organisers}

Vicky Charisi (main workshop contact) is a researcher with a special interest on the ways in which interactive and intelligent systems affect child's development. She is a researcher at the European Commission, Joint Research Centre, Centre for Advanced Studies with a focus on the impact of artificial intelligence on human behaviour. Previously, she was a post-doctoral researcher at the University of Twente, the Netherlands working on H2020 projects SQUIRREL, EASEL and DEENIGMA focusing on child-robot interaction for typically developing and autistic children.
Alyssa M. Alcorn is a postdoctoral researcher at the UCL Institute of Education, working on the Horizon 2020-funded DE-ENIGMA project (de-enigma.eu), developing a robot-assisted emotion teaching programme for autistic children aged 5-12. She received her $\mathrm{PhD}$ in human-computer interaction from the University of Edinburgh School of Informatics. Her work has focused on designing and evaluating technologies for children on the autism spectrum.

James Kennedy is a Postdoctoral Associate at Disney Research Los Angeles. His research interests lie in Human-Robot Interaction and Socially Intelligent Agents. James received his PhD from Plymouth University, U.K. in 2017 for his work using social robots to tutor children. During his PhD, he worked as a Research Assistant on the EU-funded DREAM (Development of Robot-Enhanced therapy for children with AutisM spectrum disorders) project and collaborated with the ALIZ-E (Adaptive Strategies for Sustainable Long-Term Social Interaction), and L2TOR (Second Language Tutoring using Social Robots) projects.

Wafa Johal is a postdoctoral researcher at the Computer-Human Interaction Laboratory for Learning and Instruction (CHILI) at EPFL. She obtained her PhD in Computer Sciences from the University of GrenobleAlps in France and worked on bodily signals in ChildRobot Interaction. At the CHILI Lab, she specialized on the topic of Robots for Learning, in implementing new technologies and evaluating them in classrooms or therapy centers within the CoWriter and Cellulo projects. 
Paul Baxter is a Senior Lecturer at the University of Lincoln (U.K.), in the School of Computer Science, a member of the Lincoln Centre for Autonomous Systems, and of the Autism Research and Innovation Centre. His research interests are broadly in the overlap between developmental cognitive robotics and social human-robot interaction.

Chronis Kynigos is a professor of Educational Technology and Mathematics Education and director of the Educational Technology Lab at National

Kapodistrian University of Athens. He has been involved in more than ten EC funded projects, as PI-coordinator in three. Recent example of his work is the H2020 ER4STEM project in Educational Robotics which aims to refine, unify and enhance current European approaches to STEM education through robotics in one open operational and conceptual framework.

\section{Pre-workshop Plans}

We envision this workshop bringing together researchers from academia, industry and policy making organizations, hailing from different disciplines and working on different aspects of robotics for children. Given the diverse background of the organizers of this workshop, we are planning to recruit participants from different fields through existing mailing lists (e.g. robots-worldwide); through emerging sub-communities (e.g. Working Group on Children and Robots,

International Foundation of Responsible Robotics); through existing series of workshops for educational robotics in conjunction with conferences of different fields (e.g. Robots4Learning workshop at the International Conference of Human-Robot Interaction 2018); through mailing lists of the multidisciplinary projects with which the organizers of this workshop have been involved (e.g. SQUIRREL project, DEENIGMA project, DREAM project etc.). This is in addition to publicity through IDC 2018 conference channels, and on social media. The information about our workshop will be available on the following website: https://sites.google.com/view/cri-idc-workshop.

\section{Call for Participation}

This full-day workshop aims to bring together researchers from all disciplinary backgrounds who are working on child-robot interaction (CRI), in order to reflect on the current state of this young field and to consider its 3-5 year "near future" outlook. We invite submissions (2-4 pages in SIGCHI extended abstract format) addressing any of the following topics:

- Is there (or can there be) a unified field of "childrobot interaction" across disciplines? What does that mean for future work that includes both children and robots?

- Reflections on recent or in-progress work and how it illuminates one or more current or near-future challenges for CRI as a field.

- Exploring a specific theory, methodology, or practice in current CRI research (e.g. constructivism, participatory design), and its perceived potential (or shortcomings) as a tool to meet future challenges in this area. This could include something without a current role, but with a perceived future application.

Submissions about research should include a brief overview of the project(s) and comment specifically on the disciplinary perspective(s) represented (e.g. a team with education and engineering perspectives).

Submissions on theories or methodologies should also 
briefly describe these. Papers should be submitted by April 15, 2018, via EasyChair at the following link https://easychair.org/cfp/content.cgi?a $=17240315$

All accepted position papers will be compiled into workshop proceedings, archived on arXiv. Additional resources and information are available at the workshop's webpage:

https://sites.google.com/view/cri-idc-workshop

At least one author of each accepted submission is required to attend the workshop. Participants must register for both the workshop and at least one day of the IDC conference.

\section{Acknowledgements}

We wish to thank the following EU-funded projects and their teams: ANIMATAS, DE-ENIGMA, DREAM, EASEL ER4STEM, L2TOR, SQUIRREL and the NCCR Robotics from the Swiss National Fund.

\section{References}

1. Alcorn, A. M., Tavassoli, T., Babović Dimitrijevic, S., Petrović, S., Skendzic, S., Petrović, V., \& Pellicano, E. (2017). Robots Teaching Autistic Children to Mind Read: A Feasibility Study of ChildRobot Interaction during Emotion-Recognition Training. In Innovative Technologies Demonstration Session, International Meeting for Autism Research. San Francisco, CA.

2. Alves-Oliveira, P., Arriaga, P., Paiva, A., \& Hoffman, G. (2017). YOLO, a Robot for Creativity: A Co-Design Study with Children. In Proceedings of the 2017 Conference on Interaction Design and Children (pp. 423-429). New York, NY, USA: ACM.

3. BBC News. (2018). CES 2018: Luka owl robot reads bedtime stories to kids.

4. Belpaeme, T., Kennedy, J., Baxter, P., Vogt, P., Krahmer, E., Kopp, S., ... Deblieck, T. (2015).
L2TOR - Second Language Tutoring using Social Robots. In First International Workshop on Educational Robotics at ICSR 2015.

5. Bers, M. U. (2014). Tangible kindergarten: Learning how to program robots in early childhood. In C. I. Sneider (Ed.), The Go-To Guide for Engineering Curricula PreK-5: Choosing and using the best instructional materials for your students (pp. 133-145). Thousand Oaks, CA: Corwin.

6. Charisi, V., Davison, D. P., Wijnen, F. M., Reidsma, D., \& Evers, V. (2017). Measuring Children's Perceptions of Robots' Social Competence: Design and Validation. Lecture Notes in Computer Science (Vol. 10652 LNAI).

7. Chevalier, P., Li, J. J., Ainger, E., Alcorn, A. M. Babovic, S., Charisi, V., ... Evers, V. (2017). Dialogue Design for a Robot-Based Face-Mirroring Game to Engage Autistic Children with Emotional Expressions. Lecture Notes in Computer Science (Vol. 10652 LNAI).

8. Department for Education (2013) The National Curriculum in England: Key Stages 1 and 2 framework

document. Available at: https://www.gov.uk/gover nment/publications/national-curriculum-in-englandprimary-curriculum (Accessed: 22 January 2018).

9. Fink, J., Lemaignan, S., Dillenbourg, P., Rétornaz, P., Vaussard, F., Berthoud, A., ... \& Franinović, K. (2014, March). Which robot behavior can motivate children to tidy up their toys? Design and evaluation of ranger. In Proceedings of the 2014 ACM/IEEE international conference on Human-robot interaction (pp. 439-446). ACM.

10. Gourlet, P., Le Goc, M., \& Follmer, S. (2017). Revisiting Turtles and Termites: An Open-ended Interactive Physical Game with Multiple Robots. In Proceedings of the 2017 Conference on Interaction Design and Children (pp. 679-682). New York, NY USA: ACM. 
11. Huijnen, C. A. G. J., Lexis, M. A. S., Jansens, R., \& de Witte, L. P. (2017). How to Implement Robots in Interventions for Children with Autism? A Cocreation Study Involving People with Autism, Parents and Professionals. Journal of Autism and Developmental Disorders, 47(10), 3079-3096.

12. Holloway, D., Green L. (2016). The Internet of toys. Journal of Communication Research and Practice, 2(4), 506-519.

13. Kanda, T., Sato, R., Saiwaki, N., \& Ishiguro, H. (2007). A Two-Month Field Trial in an Elementary School for Long-Term Human-Robot Interaction. Robotics, IEEE Transactions on, 23(5), 962-971.

14. Kynigos, C., Grizioti, M., \& Nikitopoulou, S. (2017). RobIn: A Half-baked Robot for Electronics in a STEM Context. In Proceedings of the 2017 Conference on Interaction Design and Children ( $\mathrm{pp}$. 521-526). New York, NY, USA: ACM.

15. Leite, I., Pereira, A., \& Lehman, J. F. (2017). Persistent Memory in Repeated Child-Robot Conversations. In Proceedings of the 2017 Conference on Interaction Design and Children ( $\mathrm{pp}$ 238-247). New York, NY, USA: ACM.

16. Ozgur, A., Johal, W., Mondada, F., \& Dillenbourg, P. (2017). Windfield: Learning Wind Meteorology with Handheld Haptic Robots. In HRI'17: ACM/IEEE International Conference on Human-Robot Interaction Proceedings (No. EPFL-CONF-224130, pp. 156-165). ACM.

17. Reidsma, D., Charisi, V., Davison, D., Wijnen, F., van der Meij, J., Evers, V., ... Verschure, P. (2016). The EASEL project: Towards educational humanrobot symbiotic interaction. Lecture Notes in Computer Science (Vol. 9793).

18. Rusk, N., Resnick, M., Berg, R., \& PezallaGranlund, M. (2008). New Pathways into Robotics: Strategies for Broadening Participation. Journal of Science Education and Technology, 17(1), 59-69.
19. Serholt, S., Barendregt, W., Vasalou, A., AlvesOliveira, P., Jones, A., Petisca, S., \& Paiva, A. (2017). The case of classroom robots: teachers' deliberations on the ethical tensions. $\{A I\}$ Soc., 32(4), 613-631.

20. Shic, F., Chawarska, K., Bradshaw, J., \& Scassellati, B. (2009). Progressive Dynamic Analysis of Face Scanning in 2- and 4-year Old Children with Autism Spectrum Disorder. In Proceedings of the 2009 Biennial Meeting for the Society for Research in Child Development (SRCD 2009).

21. Sullivan, A., Elkin, M., \& Umaschi Bers, M. U. (2015). KIBO robot demo: engaging young children in programming and engineering. Proceedings of the 14th International Conference on Interaction Design and Children. Boston, Massachusetts: ACM.

22. Sullivan, A., \& Umaschi Bers, M. U. (2015). Robotics in the early childhood classroom: learning outcomes from an 8-week robotics curriculum in pre-kindergarten through second grade. International Journal of Technology and Design Education.

23. Werry, I., \& Dautenhahn, K. (1999). Applying Mobile Robot Technology to the Rehabilitation of Autistic Children. In SIRS99, 7th Symp on Intelligent Robotic Systems (pp. 265-272).

24. Westlund, J. K., Jeong, S., Park, H., Ronfard, S., Adhikari, A., Harris, P., ... Breazeal, C. (2017). Flat vs. Expressive Storytelling: Young Children's Learning and Retention of a Social Robot's Narrative. Frontiers in Human Neuroscience, 11. 